\title{
The structure of the environment and the complexity of rational choice procedures
}

\author{
Paulo Oliva and Philipp Zahn
}

September 20, 2018

\begin{abstract}
Beginning with Herbert Simon [10], the literature on bounded rationality has investigated in great detail how internal limitations affect an agent's choice process. The structure of the choice environment, deemed as important as internal limitations by Simon [11], has been mostly ignored. We introduce a model of the environment and its interaction with an agent's choice process. Focusing on online environments where an agent can use filter and sort functionality to support his decision-making, we show, a choice process relying on the environment can be rationalized. Moreover, for sufficiently many alternatives, filtering and sorting are quick ways to choose rationally.
\end{abstract}

\section{Introduction}

In 1955 and 1956 Herbert Simon published two influential articles on individual decision-making [10, 11]. He criticized the rational choice approach dominant in economics as it ignored the actual decision making process. His critique had two dimensions: First, individuals are subject to internal limitations which shape their decision-making process. Computational capacity and memory, for instance, are constrained and have to be considered to explain choices [10]. Second, individuals are subject to external limitations which shape their decision-making process. Agents do not make decisions in the void but are embedded in an "environment" which contains information and which affects their choice procedure [11]. In particular, if the environment is sufficiently rich in relevant information, procedures can be simple but still achieve complex goals.

The first dimension has been addressed in the literature on bounded rationality and in the literature on search behavior. In this paper we focus on the second dimension which has been mostly ignored ([12] is the exception which we will discuss below). We study the interplay between the environment and the choice process of an agent in a formal model.

What is the "environment"? Broadly speaking, information relevant for an individual's choice process, in particular his search behavior. That includes information on alternatives - which we consider in the classical approach as well - but in particular information shared across alternatives. An example may help: Consider buying an external hard drive, and say you are interested in the capacity of different alternatives. Suppose you know the alternatives you consider are ordered according to their capacity. This piece of overarching information is part of the environment in our model. Online shops do not only display information, they also offer functionality to ease their customers' decision-making. Some functionality is very wide-spread across different platforms. For instance, Filtering and Sorting according to some attribute are ubiquitous. The functionality is widely used, affects choice [2], and is part the choice environment.

In this paper we consider a simple model of the environment which follows the idea of an online platform. It provides information on attributes of goods and offers filter and sort functionality in order to simplify the decision-making process of a customer. Our model shows, as noted by Simon, a sufficiently rich environment can lead to simpler procedures to achieve a complex goal function. What goal function do we consider? Rational preferences! This may be surprising because in [10] as well as the ensuing literature on bounded rationality, the main message analyzing the individual in isolation is: choosing rationally is hard. But 
how hard it really is, depends on the environment. We maintain throughout that a customer has a rational preference relation and so we exclude any other channel through which such systems may play a role. In particular, we ignore fundamental limitations on the agent's side but only maintain that his goal is to choose optimally in an as "simple" way as possible (which will be made precise). ${ }^{1}$

We show, a combination of filtering and sorting and then choosing the first element of the resulting list implements a choice which is equivalent to the optimal choice according to a preference relation defined on the attributes of goods. Preference relations which can be expressed through this operation have a simple logical structure reminiscent of the structure discussed in [7]. Such preferences can be described in a natural way. As an example, an agent expressing his preferences for an external hard drive could state the following: "I prefer hard drives with bigger capacity and prefer the Seagate brand over other brands".

Of course, it is not clear why individuals should hold such preferences. Hence, we consider two extensions. First, if preferences are defined on attributes but a particular attribute is not displayed, a combination of filtering, sorting and satisficing according to the missing attribute lead to the optimal choice. For large number of alternatives, using filter and sort functionality will lead to quicker choices than performing a full maximization on all alternatives. Secondly, we show how extending the filter functionality, by adding a union operation ("look for houses with either 5 bedrooms or located in neighborhood x"), will substantially increase the expressiveness of the preferences that our environment allows for. Doing so pushes more complexity away from the agent into the environment. If extended in such a way, for a larger set of preference relations the use of an online tool will be a quick and simple way to come to a rational decision.

Lastly, the environment not only simplifies rational choice but also other goal functions. We show, filtering can be used to implement a satisficing procedure with very few steps.

We do not claim novelty for arguing that the environment is important. But to the best of our knowledge this paper provides the first formal model where the interaction between individual procedure and the environment it faces is analyzed. Our results point to the importance of including the environment: Choosing rationally is typically hard but less so if agents' preferences have a particular form and the environment is supportive.

Relation to the Literature The paper by Todd and Girgerenzer [12] is closest in spirit: They also recognize the lack of considering the environment in the choice process. While sharing this point, our paper otherwise has little in common. We focus on a formal treatment and rational choice for a limited environment to illustrate the importance of considering the environment; they use an informal treatment and focus on simple heuristics over a broad set of different environments.

Our paper is related to the literature on search behavior, $[1,5]$ among others. These papers investigate the search process of an agent and how it relates to his underlying preferences. Our approach differs as the environment is not just providing passive information, such as an order of alternatives or a recommended element based on some statistic, but an agent can make use of functionality provided through the environment.

Our paper is related to two papers by Ariel Rubinstein and Yuval Salant $[8,9]$. In both papers, they consider decision problems with extra structure that can be used by an agent's choice procedure and then ask under which conditions such a procedure can be rationalized. In [8] they consider the case where alternatives are organized as lists; in [9] they consider additional information captured in "frames" which affects the decision-maker's choice. Possible frames include the representation of alternatives in the form of a list. In this paper, alternatives are also organized in lists and this also plays a key role in determining choices. While in their case operations on lists are internal, we consider external functionality provided from outside in addition to the representation of alternatives in lists.

Mandler et al. [4] show how a checklist approach can be seen as a procedure that is equivalent to choices made by the maximization of a utility function. The filtering functionality we consider does share resemblance with a checklist - only that in our paper it is not internal to the agent but it is provided by the environment and used by the agent to make his choice. As it is imposed from outside, and the agent cannot control the attributes that are used, the agent is constrained in the use of the functionality. Hence, not all utility functions will be implemented by it. Building on previous work [3] argues that checklists are a quick

\footnotetext{
${ }^{1}$ Adding limitations on the agent's side would reinforce our results without adding much to understanding the channel we are interested in.
} 
way to choose. We substantiate this argument by showing that filtering and sorting are typical quick ways to choose rationally and very quick ways to satisfice.

Lastly, our characterization of preference relations has similarities to [7]. Rubinstein investigates which preference relations can be expressed through the conjunction of atomic formulas or negated atomic formulas. In our case we are interested in the preference relations which can be expressed through the functionality offered by the environment. So, we do not consider all relations which can be expressed through conjunction of atomic statements but only those which are preference relations and are equivalent to procedures based on filtering and sorting.

This paper is building on results in our previous paper [6]. In that paper we introduce a general model of the environment which contains information regarding alternatives. We focus on the representational aspect of alternatives, whether agents face a list, a tree, a tree of lists etc. Using general representations, we characterize which representations and choice procedures can be rationalized. The environment we model is passive, there is no functionality an agent can employ. The paper also contains an in-depth discussion of the language we use to model representations. The fact that filtering and sorting can be rationalized in principle is a consequence of results contained in that paper. However, the results are not constructive, i.e. we show that a rational preference relation exists without characterizing it. We also do not consider the complexity of decision procedures. Lastly, in the present paper we keep the technical necessities at a minimum, for instance, we only consider one representation of alternatives in the form of lists.

\section{The Structure of the Environment}

Let us first informally state the scenario which we are interested in and then turn to the nuts and bolts. Picture a customer who wants to buy a certain type of good $x$ on an online platform. He has rational preferences and, after searching a platform, such as Amazon or Ebay, for the good, faces a list of results. The platform gives him a list of $n$ results and offers him decision support by showing attributes according to which he can filter the results as well as criteria to sort the results.

Our first task is to describe the environment and the functionality offered by it.

Definition 2.1 (Attribute) Let $X$ denote the overall set of alternatives and $(V, \succeq)$ be a totally ordered set. We call a function $a: X \rightarrow V$ an attribute map or simply an attribute.

For instance, if we take $X$ as a set of books, and model prices using positive reals with the standard ordering $\left(\mathbb{R}^{+}, \geq\right)$, we can think of a mapping price: $X \rightarrow \mathbb{R}^{+}$as assigning prices to each of the books in $X$. Moreover, if we model average review rating using the real interval [1,5] (again with the standard ordering), we can also think of the mapping rating: $X \rightarrow[1,5]$ as determining the average review $a(x) \in[1,5]$ of each given book $x \in X$, assuming customers will rate books using values $\{1,2,3,4,5\}$.

Remark 2.2 In the example above, attributes describe simple properties. We call such attributes atomic. We note here, attributes can be more complex. Consider a family looking for a holiday home. They look for a quite place, with garden and pool, and easily accessible by car. A platform may offer a composite attribute, "family friendly", which combines existing attributes in a particular way.

In this paper the decision problem is given to the agent not as a set, but as a list of alternatives []. If $X$ is the overall set of alternatives, we write List $X$ for the set of finite lists over $X$.

Definition 2.3 (Filtering) Given any attribute map $a: X \rightarrow V$ and $a$ value $v \in V$, the a-higher-than- $v$ filter function $\mathcal{F}_{a \geq v}$ : List $X \rightarrow$ List $X$ is the function which filters the elements of a list according to the predicate $a(x) \geq v$. The output list $l^{\prime}=\mathcal{F}_{a \geq v}(l)$ should satisfy the following properties:

$\left(F_{1}\right) l^{\prime}$ consists precisely of the elements $x$ of $l$ which satisfy $a(x) \geq v$.

$\left(F_{2}\right)$ if two elements $l_{i}$ and $l_{j}$ satisfy the filtering property and $i<j$ then the order of these two elements is preserved in $l^{\prime}$. 
The two conditions together ensure this uniquely defines the function $\mathcal{F}_{a \geq v}$. Dually, we can also define the $a$-lower-than- $v$-filter function $\mathcal{F}_{a \leq v}$ : List $X \rightarrow$ List $X$ satisfying similar properties.

So, for instance, we can talk about the price-lower-than-10-filter $\mathcal{F}_{\text {price } \leq 10}$. It selects only the alternatives which cost 10 or less. Or we can talk about the rating-higher-than-3-filter $\mathcal{F}_{\text {rating } \geq 3}$, which selects the alternatives with an average customer review higher than 3 .

Definition 2.4 (Sorting) Given any attribute map $a: X \rightarrow V$ we define the increasing ordering based on $a$ (also called the increasing- $a$-ordering) as the mapping $\mathcal{S}_{a}^{i}$ : List $X \rightarrow$ List $X$ which sorts the elements of a list in increasing order according to the value $a(x) \in V$. The list $l^{\prime}=\mathcal{S}_{a}^{i}(l)$ should satisfy the following three properties:

$\left(S_{1}\right) l^{\prime}$ be a permutation of the list $l$,

$\left(S_{2}\right)$ for $i<j$ we must have $a\left(l_{i}^{\prime}\right) \leq a\left(l_{j}^{\prime}\right)$, and

$\left(S_{3}\right)$ if two elements $l_{i}$ and $l_{j}$ are such that $a\left(l_{i}\right)=a\left(l_{j}\right)$ and $i<j$ then the order of these two elements is preserved in $l^{\prime}$.

These three properties uniquely define $\mathcal{S}_{a}^{i}$. Analogously we can also talk about the decreasing ordering based on $a$ (or the decreasing- $a$-ordering), i.e. $\mathcal{S}_{a}^{d}$ : List $X \rightarrow \operatorname{List} X$.

For instance, we can talk about the increasing-price-ordering or the decreasing-rating-ordering maps. Our first goal is to study the composition of these sort and filter operations.

Remark 2.5 For this paper we are assuming that the value sets $(V, \geq)$ are totally ordered sets, so that both the filtering and the sorting operations are well defined. Although filtering would technically only required $(V, \geq)$ to be a partial order, it is simpler to assume that both filtering and sorting make use of the same ordered value set. We note that there are cases where no such (natural) total ordering exists. For instance, suppose $V$ is a set of brands, e.g. $V=\{$ Toshiba, Seagate, Samsung $\}$. In these cases one can still treat these within our framework by imposing any fixed total ordering on these, e.g. lexicographical ordering. This would allow an agent to filter a particular brand by composing $\mathcal{F}_{a \leq v}$ and $\mathcal{F}_{a \geq v}$.

Definition 2.6 (Decision procedures) We will refer to the composition of different filtering and sorting operations as a decision procedure. The number of filter and sort operations used will be called the length of the procedure.

Although sorting and filtering alone will not produce a single choice at the end, but a (possibly smaller) ordered list, they form the core of how people choose online. For example, an agent first filters items with acceptable levels of average customer review, and then picks the cheapest element. Assuming an operation which picks the first element of a list, i.e.

$$
\text { first: List } X \rightarrow X,
$$

the procedure above can be described by the following composition

$$
\text { first } \circ \mathcal{S}_{\text {price }}^{i} \circ \mathcal{F}_{\text {rating } \geq 3} \quad: \quad \text { List } X \rightarrow X .
$$

This composition of operations selects a cheapest item (not necessarily unique) which has an average customer review of 3 or more. When the filtering is too restrictive, an empty list results. For now, we will not worry about this problem. We come back to it later.

Given a fixed set of attributes $\mathcal{A}$, let us define a notion of "equivalence" between decision procedures. We will also consider the equivalence of alternatives and lists - clearly prerequisites for equivalence of decision procedures.

Definition 2.7 (A-equivalence) Fix a set of attributes $\mathcal{A}$ : 
- Two alternatives $x, y \in X$ are $\mathcal{A}$-equivalent, written $x \simeq_{\mathcal{A}} y$, if $a(x)=a(y)$ for all attributes a $\in \mathcal{A}$.

- Two lists $l, l^{\prime} \in \operatorname{List} X$ are $\mathcal{A}$-equivalent, written $l \simeq_{\mathcal{A}} l^{\prime}$, if they have the same length and their corresponding elements are $\mathcal{A}$-equivalent. It is easy to see that $\simeq_{\mathcal{A}}$ is an equivalence relation on List $X$ and we will write $\llbracket l \rrbracket_{\mathcal{A}}$ for the equivalence class of $l \in$ List $X$.

- Two procedures $P_{1}, P_{2}$ : List $X \rightarrow$ List $X$ are $\mathcal{A}$-equivalent, written $P_{1} \simeq_{\mathcal{A}} P_{2}$, if for all lists $l \in$ List $X$ we have that $P_{1}(l) \simeq_{\mathcal{A}} P_{2}(l)$.

We can derive two immediate lemmas.

Lemma 2.8 Let $P, P_{1}, P_{2}$ be decision procedures defined over the set of attributes $\mathcal{A}$ :

(i) If $l_{1} \simeq_{\mathcal{A}} l_{2}$, then $P\left(l_{1}\right) \simeq_{\mathcal{A}} P\left(l_{2}\right)$.

(ii) If $l_{1} \simeq_{\mathcal{A}} l_{2}$ and $P_{1} \simeq_{\mathcal{A}} P_{2}$ then $P_{1}\left(l_{1}\right) \simeq_{\mathcal{A}} P_{2}\left(l_{2}\right)$.

Proof. ( $i$ ) By induction on the structure of $P$. If $P$ is one of the basic operations (filter or sorting) the result is obvious, e.g. clearly $\mathcal{F}_{a \geq v}\left(l_{1}\right) \simeq_{\mathcal{A}} \mathcal{F}_{a>v}\left(l_{2}\right)$ and $\mathcal{S}_{a}^{i}\left(l_{1}\right) \simeq_{\mathcal{A}} \mathcal{S}_{a}^{i}\left(l_{2}\right)$ when $l_{1} \simeq_{\mathcal{A}} l_{2}$. Now, if $P=P_{1} \circ P_{2}$, by induction hypothesis we have $P_{1}\left(P_{2}\left(l_{1}\right)\right) \simeq_{\mathcal{A}} P_{1}\left(P_{2}\left(l_{2}\right)\right)$, given $l_{1} \simeq_{\mathcal{A}} l_{2}$.

(ii) Follows directly from $(i)$ and the definition of $P_{1} \simeq_{\mathcal{A}} P_{2}$ as $P_{1}\left(l_{1}\right) \simeq_{\mathcal{A}} P_{1}\left(l_{2}\right) \simeq_{\mathcal{A}} P_{2}\left(l_{2}\right)$.

Given any attribute $a \in \mathcal{A}$, let us write $x \succeq_{a} y$ for $a(x) \geq a(y)$, and $x \preceq_{a} y$ for $a(x) \leq a(y)$. We will use $\phi, \phi_{1}, \phi_{2}, \ldots$ as meta-variables ranging over the predicates $a \geq v$ or $a \leq v$. We start by investigating some algebraic properties of composition of decision procedures with respect to this equivalence relation:

Proposition 2.9 Let $\mathcal{A}$ be a given set of attributes, and $a, a_{1}, a_{2} \in \mathcal{A}$, and $v, v_{1}, v_{2} \in V$. We have that

(i) $\mathcal{F}_{\phi_{1}} \circ \mathcal{F}_{\phi_{2}} \simeq \mathcal{F}_{\phi_{2}} \circ \mathcal{F}_{\phi_{1}}$, for all $\phi_{1}, \phi_{2} \in\left\{a_{1} \geq v_{1}, a_{2} \geq v_{2}, a_{3} \leq v_{3}, a_{4} \leq v_{4}\right\}$

(ii) $\mathcal{F}_{a \geq v_{1}} \circ \mathcal{F}_{a \geq v_{2}} \simeq \mathcal{A} \mathcal{F}_{a \geq \max \left\{v_{1}, v_{2}\right\}}$ and $\mathcal{F}_{a \leq v_{1}} \circ \mathcal{F}_{a \leq v_{2}} \simeq_{\mathcal{A}} \mathcal{F}_{a \leq \min \left\{v_{1}, v_{2}\right\}}$

(iii) $\mathcal{F}_{\phi} \circ \mathcal{S}_{a}^{\alpha} \simeq_{\mathcal{A}} \mathcal{S}_{a}^{\alpha} \circ \mathcal{F}_{\phi}$, for $\alpha \in\{i, d\}$ and $\phi \in\left\{a_{1} \geq v, a_{1} \leq v\right\}$.

(iv) $P_{1} \simeq_{\mathcal{A}} P_{2}$ and $P_{1}^{\prime} \simeq_{\mathcal{A}} P_{2}^{\prime}$ then $P_{1} \circ P_{1}^{\prime} \simeq_{\mathcal{A}} P_{2} \circ P_{2}^{\prime}$, for arbitrary procedures $P_{1}, P_{1}^{\prime}, P_{2}, P_{2}^{\prime}$

(v) $\mathcal{S}_{a}^{\alpha} \circ P \circ \mathcal{S}_{a}^{\beta} \simeq_{\mathcal{A}} \mathcal{S}_{a}^{\alpha} \circ P$, for $\alpha, \beta \in\{i, d\}$ and $P$ an arbitrary procedure

Proof. (i) Clearly the order in which one applies two filters to a list does not matter. The two resulting lists will actually be identical, and hence $\mathcal{A}$-equivalent for any attribute set $\mathcal{A}$.

(ii) Easy. Note that $\mathcal{F}_{a \geq v_{1}} \circ \mathcal{F}_{a \leq v_{2}}$, however, cannot in general be simplified as it is specifying a genuine interval of preference - except when $v_{2}<v_{1}$ in which case this would always return the empty list.

(iii) Consider the case when $\alpha=i$ and $\phi=a_{1} \geq v$, the other case is handled similarly. Let $l \in$ List $X$ and assume $l=\mathcal{F}_{a_{1} \geq v}\left(\mathcal{S}_{a}^{i}(l)\right)$ and $l^{\prime}=\mathcal{S}_{a}^{i}\left(\mathcal{F}_{a_{1} \geq v}(l)\right)$. Again, it is easy to see that in this case $l=l^{\prime}$, and in particular they are $\mathcal{A}$-equivalent.

(iv) Easy consequence of Lemma 2.8. Indeed, given a list $l$, by the assumption $P_{1}^{\prime} \simeq_{\mathcal{A}} P_{2}^{\prime}$ we have $P_{1}^{\prime}(l) \simeq_{\mathcal{A}}$ $P_{2}^{\prime}(l)$. By Lemma $2.8(i i)$ and the assumption $P_{1} \simeq_{\mathcal{A}} P_{2}$ we obtain $P_{1}\left(P_{1}^{\prime}(l)\right) \simeq_{\mathcal{A}} P_{2}\left(P_{2}^{\prime}(l)\right)$.

$(v)$ We are claiming here that sorting with respect to an attribute $a$, and then later sorting with respect to the same attribute (possibly in the opposite direction) makes the initial sorting irrelevant. By $(i),(i i i)$ and $(i v)$, we can assume that $P$ only contains sorting operations, i.e. we must show

$$
\mathcal{S}_{a}^{\alpha} \circ \mathcal{S}_{a_{1}}^{\alpha_{1}} \circ \ldots \circ \mathcal{S}_{a_{n}}^{\alpha_{n}} \circ \mathcal{S}_{a}^{\beta} \simeq_{\mathcal{A}} \mathcal{S}_{a}^{\alpha} \circ \mathcal{S}_{a_{1}}^{\alpha_{1}} \circ \ldots \circ \mathcal{S}_{a_{n}}^{\alpha_{n}}
$$

Moreover, by induction we can assume that all $a_{i}$ are different from $a$. Now, one can see that the procedure $P \equiv \mathcal{S}_{a_{1}}^{\alpha_{1}} \circ \ldots \mathcal{S}_{a_{n}}^{\alpha_{n}}$ is ordering the elements of any given list according to the lexicographical ordering on the relations $R_{i} \in\left\{\preceq_{a_{i}}, \succeq_{a_{i}}\right\}$ depending on whether $\alpha_{i}$ is $i$ or $d$. In the case $\mathcal{S}_{a}^{\alpha} \circ P$ we are sorting by $a$, and 
using the lexicographical ordering of $P$ for attributes that have the same $a$ value. In the case of $\mathcal{S}_{a}^{\alpha} \circ P \circ \mathcal{S}_{a}^{\beta}$ one is also first sorting by $a$, and then by the lexicographical ordering of $P$ for elements that have the same $a$ value, and finally sorting by $a$ again in case of tie-break. But this final step is indeed redundant since we will only apply these to elements which already have the same $a$ value.

Equipped with the proposition before, we can now associate with any decision procedure a canonical equivalent which minimizes the number of steps needed.

Theorem 2.10 (Normal form) We say that two filtering operations $\mathcal{F}_{\phi_{1}}$ and $\mathcal{F}_{\phi_{2}}$ are dual if $\phi_{1}=a \geq v_{1}$ and $\phi_{2}=a \leq v_{2}$. Any decision procedure is $\mathcal{A}$-equivalent to a procedure where:

1. all filtering operations precede all sorting operations,

2. all sorting operations are done with respect to distinct attributes, and

3. at most two dual filtering operations are used for each attribute.

Proof. Straightforward from Proposition 2.9.

As we can derive for each procedure its normal form, we can also derive a minimal number of steps needed to compute a choice.

Corollary 2.11 Let $N=|\mathcal{A}|$. Any decision procedure is $\mathcal{A}$-equivalent to a procedure of length at most $3 N$.

For instance, the decision procedure

$$
\mathcal{S}_{\text {rating }}^{d} \circ \mathcal{S}_{\text {price }}^{i} \circ \mathcal{F}_{\text {rating } \geq 4} \circ \mathcal{S}_{\text {price }}^{d}
$$

is $\{$ price, rating $\}$-equivalent to

$$
\mathcal{S}_{\text {rating }}^{d} \circ \mathcal{S}_{\text {price }}^{i} \circ \mathcal{F}_{\text {rating } \geq 4}
$$

which indicates preference for the cheapest highest-rated product amongst these with a rating higher-than 4 . We consider this to be the normal form of the procedure above, and this normal form procedure has length 3. In summary, for all procedures we can derive a canonical normal form. Moreover, note that if we fix an ordering on the set of attributes $\mathcal{A}$, and put all filtering operations in this order, the normal form of the procedure will be unique. This result will become handy in the next section when we characterize what type of preferences can result from sorting and filtering.

\section{Rationalizable Preference Relations}

So far, we described the environment and decision procedures operating on it. We also characterized these procedures through its normal form. We want to relate procedures to the maximization of a rational preference relation.

We begin by characterizing preferences in our setting. A preference relation $\succeq$ is a subset of $X \times X$ satisfying the two rationality conditions: completeness (either $x \succeq y$ or $y \succeq x$ ) and transitivity ( $x \succeq y$ and $y \succeq z$ implies $x \succeq z$ ). When $x \succeq y$ we say that $x$ is weakly preferred over $y$. One is indifferent between $x$ and $y$ when $x \succeq y$ and $y \succeq x$, which is written as $x \sim y$. If $x$ is weakly preferred over $y$ but we are not indifferent between the two then we also say that $x$ is strictly preferred over $y$, written as $x \succ y$.

We will see the type of preferences which can be expressed through filtering and sorting is restricted. To characterize these restrictions, we first introduce some properties.

Definition 3.1 (A-definable property) $A$ subset of $X$ is said to be $\mathcal{A}$-definable if it is a conjunction of atomic statements such as $a(x) \geq p$ and $a(x) \leq p$, where $a \in \mathcal{A}$ and $p \in P$.

For instance, the set of alternatives which are cheaper than $\$ 10$ but have an average rating higher than 3 is $\{$ price, $\operatorname{rating}\}$-definable as $\operatorname{price}(x) \leq 10 \wedge \operatorname{rating}(x) \geq 3$. 
Definition $3.2(\mathcal{A}$-definable ordering) An ordering on $X$ is said to be $\mathcal{A}$-definable if it is the lexicographical combination of the orderings $\succeq_{a}$ and $\preceq_{a}$ for $a \in \mathcal{A}$.

For instance, the ordering on $X$ which first orders elements by increasing price order and then by decreasing rating order (when two elements have the same price) is an $\mathcal{A}$-definable ordering. When talking about $\mathcal{A}$-definable ordering we will use the notation $\succeq_{\left(\alpha_{1}, a_{1}\right) \times \ldots \times\left(\alpha_{n}, a_{n}\right)}$ where $\alpha_{i} \in\{i, d\}$ and $a_{i} \in \mathcal{A}$, denoting the ordering which first considers the $\alpha_{1}$-direction of property $a_{1}$, then the $\alpha_{2}$-direction of property $a_{2}$ and so on. In this way, the ordering above can be written concisely as $\succeq_{(i, \text { price }) \times(d, \text { rating })}$.

Definition 3.3 ( $\mathcal{A}$-definable preference relation) $A$ preference relation $A \subseteq X \times X$ is said to be $\mathcal{A}$ definable if it can be expressed as $A(x, y) \equiv \neg F(y) \vee(F(x) \wedge x \succeq y)$ where $F$ is an $\mathcal{A}$-definable property and $\succeq$ is an $\mathcal{A}$-definable ordering.

Proposition 3.4 For any $\mathcal{A}$-definable property $F$ and any $\mathcal{A}$-definable ordering $\succeq$ the relation

$$
\neg F(y) \vee(F(x) \wedge x \succeq y)
$$

is a preference relation.

Proof. Fix an $\mathcal{A}$-definable filter $F$ and $\mathcal{A}$-definable ordering $\succeq_{\left(\alpha_{1}, a_{1}\right) \times \ldots \times\left(\alpha_{n}, a_{n}\right)}$; and let

$$
x \succeq y \equiv \neg F(y) \vee\left(F(x) \wedge x \succeq_{\left(\alpha_{1}, a_{1}\right) \times \ldots \times\left(\alpha_{n}, a_{n}\right)} y\right)
$$

Let us first show that either $x \succeq y$ or $y \succeq x$. Indeed, for both of these to fail we should have $F(x)$ and $F(y)$ but not $x \succeq_{\left(\alpha_{1}, a_{1}\right) \times \ldots \times\left(\alpha_{n}, a_{n}\right)} y$ or $y \succeq_{\left(\alpha_{1}, a_{1}\right) \times \ldots \times\left(\alpha_{n}, a_{n}\right)} \quad x$, which is impossible since the completeness of each individual ordering $\succeq_{\left(\alpha_{i}, a_{i}\right)}$ implies the completeness of the lexicographical ordering $\succeq_{\left(\alpha_{1}, a_{1}\right) \times \ldots \times\left(\alpha_{n}, a_{n}\right)}$. In order to show the transitivity of $\succeq$, assume $x \succeq y$ and $y \succeq z$. If $\neg F(z)$ we are done. Hence, suppose $F(z)$, and we must argue that $F(x)$ and $x \succeq_{\left(\alpha_{1}, a_{1}\right) \times \ldots \times\left(\alpha_{n}, a_{n}\right)} z$. Indeed, $F(z)$ and $y \succeq z$ gives us $F(y)$ and $y \succeq_{\left(\alpha_{1}, a_{1}\right) \times \ldots \times\left(\alpha_{n}, a_{n}\right)} z$, while $F(y)$ and $x \succeq y$ gives us $F(x)$ and $x \succeq_{\left(\alpha_{1}, a_{1}\right) \times \ldots \times\left(\alpha_{n}, a_{n}\right)} y$. These indeed imply $x \succeq_{\left(\alpha_{1}, a_{1}\right) \times \ldots \times\left(\alpha_{n}, a_{n}\right)} y$ by the transitivity of each individual ordering $\succeq_{\left(\alpha_{i}, a_{i}\right)}$.

Definition 3.5 (Preference relation derived from a procedure) Given a decision procedure $P$ : List $X \rightarrow$ List $X$ its preference relation is defined as

$$
x \succeq_{P} y \equiv \exists l \in \text { List } X\left(x, y \in l \wedge l^{\prime}=P(l) \wedge \forall j\left(y=l_{j}^{\prime} \rightarrow \exists i<j\left(x=l_{i}^{\prime}\right)\right)\right)
$$

In words, we say that $x$ is $P$-weakly preferred over $y$ if for some list containing both $x$ and $y$ we have that the procedure $P$ either filters out $y$, or it leaves both elements but ranks $x$ higher than $y$. It is not obvious that $x \succeq_{P} y$ as defined above is a preference relation. Proposition 3.4 and the following theorem show that this is indeed the case.

Theorem 3.6 (Soundness) For any procedure P: List $X \rightarrow$ List $X$ over the set of attributes $\mathcal{A}$ there exists an $\mathcal{A}$-definable preference relation $x \succeq y$ such that $x \succeq_{P} y \Leftrightarrow x \succeq y$.

Proof. By the normal form theorem we have that $P$ is equivalent to a procedure of the form

$$
\mathcal{S}_{a_{1}}^{\alpha_{1}} \circ \ldots \circ \mathcal{S}_{a_{n}}^{\alpha_{n}} \circ \mathcal{F}_{\phi_{1}} \circ \ldots \circ \mathcal{F}_{\phi_{m}}
$$

where $a_{i}$ are distinct attributes and $\alpha_{i} \in\{i, d\}$ and $\phi_{j}$ are of the form $a \geq v$ or $a \leq v$. Let $F(x)$ be the $\mathcal{A}$-definable property

$$
F(x) \equiv \bigwedge_{j=1}^{m} \phi_{j}
$$

and let $\succeq_{\left(\alpha_{1}, a_{1}\right) \times \ldots \times\left(\alpha_{n}, a_{n}\right)}$ be the $\mathcal{A}$-definable ordering. Finally, let $x \succeq y$ be the $\mathcal{A}$-definable preference relation

$$
x \succeq y \equiv \neg F(y) \vee\left(F(x) \wedge x \succeq_{\left(\alpha_{1}, a_{1}\right) \times \ldots \times\left(\alpha_{n}, a_{n}\right)} y\right)
$$


We claim that $x \succeq_{P} y \Leftrightarrow x \succeq y$.

First, assuming $x \succeq_{P} y$ holds, i.e. there exists a list $l$ such that

$$
x, y \in l \wedge l^{\prime}=P(l) \wedge \forall j\left(y=l_{j}^{\prime} \rightarrow \exists i<j\left(x=l_{i}^{\prime}\right)\right) .
$$

If $\neg F(y)$ then $x \succeq y$ holds trivially and we are done. So let us assume $F(y)$. This means that $y$ passes all the filters of $P$ and hence $y \in l^{\prime}$ and $x \in l^{\prime}$, with $y$ coming later than $x$ on the resulting list. Since the sorting component of $P$ is $\mathcal{S}_{a_{1}}^{\alpha_{1}} \circ \ldots \circ \mathcal{S}_{a_{n}}^{\alpha_{n}}$, and $y$ comes later than $x$ in $l^{\prime}$, it is clear that $x \succeq_{\left(\alpha_{1}, a_{1}\right) \times \ldots \times\left(\alpha_{n}, a_{n}\right)} y$.

For the other direction, assume $\neg F(y) \vee\left(F(x) \wedge x \succeq_{\left(\alpha_{1}, a_{1}\right) \times \ldots \times\left(\alpha_{n}, a_{n}\right)} y\right)$. We must show that for some list $l$ containing $x$ and $y$ we have either

$$
y \notin l^{\prime} \quad \text { or } \quad y=l_{j}^{\prime} \wedge x=l_{i}^{\prime} \wedge i<j
$$

where $l^{\prime}=P(l)$. If $y$ does not pass one of the filters of $P$ this is an easy task. So let us assume $F(y)$. Our assumption then gives $F(x)$ and $x \succeq_{\left(\alpha_{1}, a_{1}\right) \times \ldots \times\left(\alpha_{n}, a_{n}\right)} y$. Hence, let $l$ be any list where $x$ is listed before $y$. Since we have $F(x)$ and $F(y)$, we obtain that $x, y \in l^{\prime}$. Assume $x=l_{i}^{\prime}$ and $y=l_{j}^{\prime}$. Finally, since $x \succeq_{\left(\alpha_{1}, a_{1}\right) \times \ldots \times\left(\alpha_{n}, a_{n}\right)} y$, and the sorting operations of $P$ match this lexicographical ordering, we indeed have that $i<j$.

The converse of the above also holds, i.e. there is a one-to-one correspondence between preference relations arising from procedures and $\mathcal{A}$-definable preference relations.

Theorem 3.7 (Completeness) For any $\mathcal{A}$-definable preference relation $(X, \succeq)$ there is an $\mathcal{A}$ procedure $P_{\succeq}:$ List $X \rightarrow$ List $X$ such that $x \succeq_{P_{\succeq}} y$ iff $x \succeq y$.

Proof. Suppose the given $\mathcal{A}$-definable preference relation is of the form

$$
x \succeq y \equiv F(y) \rightarrow\left(F(x) \wedge x \succeq_{\left(\alpha_{1}, a_{1}\right) \times \ldots \times\left(\alpha_{n}, a_{n}\right)} y\right)
$$

for an $\mathcal{A}$-definable property

$$
F(x) \equiv \bigwedge_{j=1}^{m} \phi_{j}
$$

Define the procedure

$$
P_{\succeq} \equiv \mathcal{S}_{a_{1}}^{\alpha_{1}} \circ \ldots \mathcal{S}_{a_{n}}^{\alpha_{n}} \circ \mathcal{F}_{\phi_{1}} \circ \ldots \circ \mathcal{F}_{\phi_{m}}
$$

We can show that $x \succeq_{P} y$ iff $x \succeq y$ using a similar argument to the proof of Theorem 3.6. First, assuming $x \succeq y$, i.e.

$$
F(y) \rightarrow\left(F(x) \wedge x \succeq_{\left(\alpha_{1}, a_{1}\right) \times \ldots \times\left(\alpha_{n}, a_{n}\right)} y\right)
$$

we need to produce a list $l$ containing both $x$ and $y$ such that

$$
\forall j\left(y=l_{j}^{\prime} \rightarrow \exists i<j\left(x=l_{i}^{\prime}\right)\right)
$$

where $l^{\prime}=P_{\succeq}(l)$. Again, if $\neg F(y)$, meaning that $y$ does not pass the filter $F$, we can take $l$ to be any list containing $x$ and $y$, since $y$ will be filtered out and $y \notin l^{\prime}$. Assume, however, that $F(y)$, then by our assumption we must also have $F(x)$ and $x \succeq_{\left(\alpha_{1}, a_{1}\right) \times \ldots \times\left(\alpha_{n}, a_{n}\right)} y$. But this indeed implies that in any list $l$ containing $x$ and $y$, with $x$ listed before $y$, we will have (for $l^{\prime}=P(l)$ ) that $x, y \in l^{\prime}$ and $x$ is be sorted ahead of $y$ in $l^{\prime}$. The converse, that $x \succeq_{P_{\succeq}} y$ implies $x \succeq y$, also follows a similar pattern to the proof of Theorem 3.6.

To sum up: For agents who have $\mathcal{A}$-definable preferences, sorting and filtering is all they need. They can choose the first element and will get their optimal choice.

It is not only interesting that agents can use filtering and sorting to choose optimally but doing so will be simple. In order to be able to compare procedures based on sorting and filtering to other ways of optimizing we need to define a benchmark case. 
Definition 3.8 (Element-by-element optimization) The agent compares the first two elements of the list and keeps the better element. He proceeds by comparing the better element to the third element; keeping the better element again. He continues in this way to the last element.

Consider a simple but for our purpose sufficient measure of simplicity: the number of steps needed to find the optimal alternative. In the case of element-by-element optimization, or for that matter any procedure which inspects the whole list, in order to find the optimal element in a list of length $n$, the agent needs to make $n-1$ comparisons.

Proposition 3.9 Assume an agent holds $\mathcal{A}$-definable preferences and the number of attributes used by him is $N \leq|\mathcal{A}|$ and the length of the input list is $n$. If $N<(n-1) / 3$ then choosing by filtering and sorting is quicker than maximizing element by element.

Proof. The agent filters or sorts according to the attributes and then chooses the first element on the resulting list. As the number of attributes is $N$, by Corollary 2.11, the length of the procedure (in normal form) will be at most $3 N$. And if $3 N<n-1$, choosing in this way will be quicker than the element-by-element optimization.

From the last result, it should be clear that with increasing variety, the decision support by filtering and sorting is more and more likely to quicken the time needed to find the optimal element.

So far we assumed agents hold specific preferences and the platform does provide the relevant attributes. In the next section we turn to cases where one of these assumptions is violated and study in which sense the available functionality still works approximately.

\section{Dealing with Missing Attributes}

Let us consider the case when the agent's preferences are defined on a strictly larger set of attributes $\mathcal{A}^{\prime} \supset \mathcal{A}$. For instance, suppose an online shop allows customers to sort and filter with respect to price and rating, but the customer also has some preferences over the dimension (e.g. of a table) or the capacity (e.g. of an external hard disk). In this section, we discuss how the agent might still be able to use the operations available to assist in their choice.

\subsection{Less filters means more satisficing}

Consider first an agent who would like to choose the cheapest highest-rated hard disk with a capacity of at least $1 \mathrm{~GB}$, but $\mathcal{A}=$ \{price, rating $\}$. His preference can be fully captured by a procedure over the extended set of attributes $\mathcal{A}^{\prime}=\{$ price, rating, capacity $\}$, namely

$$
\mathcal{S}_{\text {rating }}^{d} \circ \mathcal{S}_{\text {price }}^{i} \circ \mathcal{F}_{\text {capacity } \geq 1 \mathrm{~GB}}
$$

Over the smaller attribute set $\mathcal{A}=\{$ price, rating $\}$, where the filtering operation $\mathcal{F}_{\text {capacity } \geq 1 \mathrm{~GB}}$ is missing, we can only approximate this procedure as

$$
\mathcal{S}_{\text {rating }}^{d} \circ \mathcal{S}_{\text {price }}^{i}
$$

What should the agent then do? He cannot filter the elements by capacity. So, he must manually inspect the list, mustn't he?

We claim: The agent can still use the available operations, namely $\mathcal{S}_{\text {rating }}^{d} \circ \mathcal{S}_{\text {price. }}^{i}$ Then, he can apply a satisficing procedure on the set capacity $\geq 1 \mathrm{~GB}$. The lack of a filtering mechanism on a particular attribute can be replaced by a final satisficing step.

Satisficing can be formally defined here as follows: 
Definition 4.1 (Satisficing) Consider an agent who has in mind a partition of the set of alternatives $X$, $\{S, X \backslash S\}$, with $S$ describing the set of alternatives which are satisfactory. A satisficing List -decision procedure consists of the agent scanning the list until a satisfactory element is found. If none is found the last element is chosen. This procedure can be described by a simple recursive procedure as:

$$
\begin{aligned}
& \xi_{S}([x])=x \\
& \xi_{S}(x: x s)=\text { if } x \in S \text { then } x \text { else } \xi_{S}(x s)
\end{aligned}
$$

The lack of a filter operation can be replaced by a final satisficing step:

Proposition 4.2 For any List-decision procedure $P$ we have that

$$
\left(\text { first } \circ P \circ \mathcal{F}_{a \geq v}\right)(l)=\left(\xi_{\{x: a(x) \geq v\}} \circ P\right)(l)
$$

for any list $l$ for which $\left(P \circ \mathcal{F}_{a \geq v}\right)(l)$ is not empty.

Proof. Fix $l \in$ List $X$ and assume $\left(P \circ \mathcal{F}_{a \geq v}\right)(l)$ is not empty, so that $P(l)$ is not empty either. By Proposition $2.9\left(P \circ \mathcal{F}_{a \geq v}\right)(l)=\left(\mathcal{F}_{a \geq v} \circ P\right)(l)$. Hence, (first $\left.\circ P \circ \mathcal{F}_{a \geq v}\right)(l)$ is the first element of $P(l)$ which satisfies $a \geq v$. That is exactly what the satisficing procedure $\xi_{\{x: a(x) \geq v\}}$ would do to the list $P(l)$.

According to the above proposition, if the agent follows a satisficing procedure and can use the existing attributes on the platform, then filtering will produce a list of results which are all satisfactory to the agent. He then can choose any of the resulting list and will be satisfied. In other words, if an attribute is missing, the agent can use a satisficing procedure instead. Hence, after filtering with the available attributes, he can then use a satisficing procedure to find an element that satisfies the missing attribute.

In passing, we note the following relationship between satisficing, filtering, and maximization:

Proposition 4.3 If the satisficing set $S$ consists of the maximal elements with respect to an $\mathcal{A}$-definable preference relation $\succeq$, then (first $\left.\circ P_{\succeq}\right)(l)=\xi_{S}(l)$, where $P_{\succeq}$ is defined in the proof of Proposition 3.7, and assuming $l$ is such that $l_{i} \in S$ for some index $i$.

Proof. Suppose the given $\mathcal{A}$-definable preference relation is of the form

$$
x \succeq y \equiv F(y) \rightarrow\left(F(x) \wedge x \succeq_{\left(\alpha_{1}, a_{1}\right) \times \ldots \times\left(\alpha_{n}, a_{n}\right)} y\right)
$$

for an $\mathcal{A}$-definable property $F(x) \equiv \bigwedge_{j=1}^{m} \phi_{j}$ so that $P_{\succeq}$ is the procedure

$$
P_{\succeq} \equiv \mathcal{S}_{a_{1}}^{\alpha_{1}} \circ \ldots \mathcal{S}_{a_{n}}^{\alpha_{n}} \circ \mathcal{F}_{\phi_{1}} \circ \ldots \circ \mathcal{F}_{\phi_{m}}
$$

Let $l$ be a list such that $l_{i} \in S$ for some index $i$. It is clear that the first element $l_{0}^{\prime}$ of $l^{\prime}=P_{\succeq}(l)$ will be such that $F\left(l_{0}^{\prime}\right)$ and $l_{0}^{\prime}$ will be maximal in $l^{\prime}$ with respect to the order $\left(\alpha_{1}, a_{1}\right) \times \ldots \times\left(\alpha_{n}, a_{n}\right)$. It remains to see that for any other element in $l$ which also satisfies $F$ and is maximal with respect to $\left(\alpha_{1}, a_{1}\right) \times \ldots \times\left(\alpha_{n}, a_{n}\right)$, has to come at position $l_{k}^{\prime}$ for some $k>0$ by the assumption $\left(S_{3}\right)$ on the sorting operation.

That is, if an agent is happy with any of the alternatives in $S$, and if $S$ are the optimal elements according to an $\mathcal{A}$-definable preference relation, then the agent can replace the satisficing procedure by the much quicker procedure of applying $P_{\succeq}$ and then selecting the first element.

Turning back to the filter, sort, satisficing procedure, why should an agent consider a satisficing step at all? Because often it is quicker than element-by-element maximization!

Proposition 4.4 Suppose $\mathcal{A} \subset \mathcal{A}^{\prime}$. If the agent holds $\mathcal{A}^{\prime}$-definable preferences (using $N$ attributes) and $n>n^{\prime}+3 N$, where $n$ is the length of the list of alternatives, $n^{\prime}$ is the length of the list after filtering, using the filter and sort procedure available in $\mathcal{A}$, followed by satisficing on the attribute not listed is quicker than element-by-element maximization. 
Proof. The agent filters or sorts according to the attributes in $\mathcal{A}$ and then satisfices w.r.t. the missing attribute $\mathcal{A}^{\prime} \backslash \mathcal{A}$. By Corollary 2.11, each attribute is used at most three times. Hence, if the filter and sort combination sufficiently reduce the number of alternatives, here measured by the difference between the initial list $n$ and the resulting list $n^{\prime}$, then satisficing will be at least as quick as maximization of the reduced list. In all but the case where there is only one element that is satisfactory and happens to be the last element on the list, satisficing will be quicker than element-by-element maximization.

\subsection{Less sorting means more local maximization}

Let us now turn to the case where some sorting operations are missing. Consider an agent would like to choose the cheapest disk with the highest capacity. This again can be described by a procedure over the attribute set $\mathcal{A}^{\prime}=\{$ price, rating, capacity $\}$ as

$$
\mathcal{S}_{\text {capacity }}^{d} \circ \mathcal{S}_{\text {price }}^{i}
$$

but over the smaller set $\mathcal{A}=\{$ rating, capacity $\}$ all we can do is sort by capacity. How should the agent then find the cheapest disk with the highest capacity? It might seem at first that the agent would need to scan the whole list in order to find such disk. But since only the disk with highest capacity should be considered, the search for the cheapest disk is done locally only within the products with highest capacity!

Therefore, the lack of some sorting mechanism implies the need for the agent to scan some portion of the result performing a local maximization procedure.

Definition 4.5 (Local maximization) Consider an agent who would like to maximize with respect to some attribute $a$, but will only consider the initial elements that have the same $a_{1}, \ldots, a_{n}$-values for some other attributes $a_{1}, \ldots, a_{n}$. A local maximization List-decision procedure consists of the agent scanning the list until a different $a_{1}, \ldots, a_{n}$-values is found, and then taking the a-maximal element seen up to that point. This procedure can also be described by a simple recursive algorithm as:

$$
\begin{gathered}
\nu_{a ; a_{1} \ldots a_{n}}^{\prime}(b)([])=b \\
\quad \nu_{a ; a_{1} \ldots a_{n}}^{\prime}(b)(x: x s)=\text { if } \vec{a}(x)=\vec{a}(b) \text { then } \nu_{S}\left(\max _{a}\{x, b\}\right)(x s) \text { else } b \\
\text { and } \nu_{a ; a_{1} \ldots a_{n}}(x: x s)=\nu_{a ; a_{1} \ldots a_{n}}^{\prime}(x)(x s) \text {, where } \vec{a}(x)=\vec{a}(b) \text { means } a_{i}(x)=a_{i}(b) \text { for all } 1 \leq i \leq n .
\end{gathered}
$$

Proposition 4.6 For any attributes $a$ and $a^{\prime}$ and procedure $P$, we have that

$$
\left(\text { first } \circ \mathcal{S}_{a_{1}}^{\alpha_{1}} \circ \ldots \circ \mathcal{S}_{a_{n}}^{\alpha_{n}} \circ \mathcal{S}_{a}^{d} \circ P\right)(l)=\left(\nu_{a ; a_{1} \ldots a_{n}} \circ \mathcal{S}_{a_{1}}^{\alpha_{1}} \circ \ldots \circ \mathcal{S}_{a_{n}}^{\alpha_{n}} \circ P\right)(l)
$$

i.e. performing a local maximization over a on an initial segment of a list which has the same $a_{1} \ldots a_{n}$ values, is equivalent to sorting the list in decreasing a-order first, then by $a_{1} \ldots a_{n}$ order, and taking the first element.

Why should an agent do the local maximization? Because often it is the quickest way to find the best alternative!

As long as the number of alternatives is large, and preferences can be defined on attributes - even if some of them are not listed, the filtering and sorting help to choose optimally. And if the number of alternatives increases, they help even more to quickly find the best alternative.

Remark 4.7 (Approximating through filtering and sorting) What can an agent do, if his preferences cannot be directly expressed through the available functionality? He will need to scan the whole list of alternatives, if he wants to fully optimize. Depending on the number of alternatives, this can be a daunting task. In such a situation, the agent may deviate from full optimization and revert to an approximation. Obviously, there are many ways he could shortcut the problem (and many have been discussed in the literature). There is no way to generally state what an approximation should be. Note though, one prominent alternative to full optimization is the satisficing procedure introduced above. An agent following this procedure inspects the items in the list until he finds an object which is satisfactory for him. And as noted above, satisficing can be implemented very fast using filtering. 


\section{More General Procedures}

So far, we have explored the relationship between a limited online environment, the functionality offered for a customer, and what kind of preference relations can be directly expressed through this functionality. In the last section, we considered the case where the environment is not expressive enough.

Consider a property buyer is looking for the cheapest house which either has three bedrooms or is located within 1 mile from the train station. So both types of houses are substitutes for him, and he would like to find the cheapest of these. Assuming the attributes $\mathcal{A}=$ \{bedrooms, price, distance $\}$ are available online, the desired query would need to involve a union of two filtering operations. But our available composition of filters only captures the intersection operation, i.e. the operation

$$
\text { first } \circ \mathcal{S}_{\text {price }}^{i} \circ \mathcal{F}_{\text {bedrooms } \geq 3} \circ \mathcal{F}_{\text {distance } \leq 1}
$$

finds the cheapest house, which has 3 or more bedrooms and is within one mile from the train station.

At first, it might seem, the agent can simply perform two queries: one looking at houses with three bedrooms, and another looking at houses near the station. But with two lists one can no longer easily apply further operations, such as sorting the results according to price and year of construction, etc.

Note this issue would be resolved by the platform, if it offered this particular combination as a single attribute three_beds_or_near_station. Although artificial in the particular example, it might make sense to extend the attribute set with this compound attribute if it is commonly requested. More generally, the set of attributes $\mathcal{A}$ available online does not need to contain just atomic or even simple composite attributes but could also include much richer attributes. In this section, however, we go in a different direction. We extend the platform with a general union operation and consider the implications.

Definition 5.1 (General decision filter) Define the intersection and union of filter operations as

- $\mathcal{F}_{\phi_{1}} \cap \mathcal{F}_{\phi_{2}}:$ List $X \rightarrow \operatorname{List} X$ as the filter operation which will discard the elements of the list unless it satisfies both filter conditions $\phi_{1}$ and $\phi_{2}$.

- $\mathcal{F}_{\phi_{1}} \cup \mathcal{F}_{\phi_{2}}$ : List $X \rightarrow$ List $X$ as the filter operation which will discard the elements of the list unless it satisfies at least one of the filter conditions $\phi_{1}$ or $\phi_{2}$.

For each attribute $a: X \rightarrow V$ let us call the filter operations $\mathcal{F}_{a \geq v}$ and $\mathcal{F}_{a \leq v}$ atomic filters. We then call a general decision filter any combination of the atomic filters by means of unions and intersections.

For instance, we can filter out the houses that have a price tag of at most $€ 2000$ and, have 3 or more bedrooms or are within one mile from the train station, as

$$
\mathcal{F}_{\text {price } \leq 2000} \cap\left(\mathcal{F}_{\text {bedrooms } \geq 3} \cup \mathcal{F}_{\text {distance } \leq 1}\right)
$$

Proposition 5.2 Any general decision filter is equivalent to one which is the intersection of the union of atomic filters.

Proof. A basic result of propositional logic says that any propositional formula is logically equivalent to one in conjunctive normal form, i.e. it is the disjunction of conjunctions. When applied to the above, we see that it is sufficient to consider general decision filters which are obtained via the intersection of unions, and that this is enough to capture any general decision filter.

It is therefore sufficient to extend an online platform with an operation for the union of atomic filters, since the intersection is already captured by the sequential composition of procedures.

Definition 5.3 (General decision procedure) Given a set of attributes $\mathcal{A}$, the general decision procedures over $\mathcal{A}$ are those obtained by compositing sorting operations with a general decision filter.

Since our normal form theorem shows that we can, without loss of generality, assume that filtering operations are done before sorting, these general decision procedures indeed generalizes our previous notion of a decision procedure. 
Definition 5.4 (General $\mathcal{A}$-definable property) $A$ subset of $X$ is said to be general $\mathcal{A}$-definable if it can be expressed in propositional logic from the atomic predicates $a(x) \geq v$ and $a(x) \leq v$, where $a: X \rightarrow V$ is an attribute in $\mathcal{A}$ and $v \in V$.

Definition 5.5 (General $\mathcal{A}$-definable preference relation) A preference relation $A \subseteq X \times X$ is said to be general $\mathcal{A}$-definable if it can be expressed as $A(x, y) \equiv \neg F(y) \vee(F(x) \wedge x \succeq y)$ where $F$ is a general $\mathcal{A}$-definable property and $\succeq$ is an $\mathcal{A}$-definable ordering.

Theorem 5.6 (General soundness) For any general procedure $P:$ List $X \rightarrow \operatorname{List} X$ over the set of attributes $\mathcal{A}$ there exists a general $\mathcal{A}$-definable preference relation $x \succeq y$ such that $x \succeq y \Leftrightarrow x \succeq_{P} y$.

Proof. A general produce $P$ : List $X \rightarrow$ List $X$ is of the form

$$
\mathcal{S}_{a_{1}}^{\alpha_{1}} \circ \ldots \circ \mathcal{S}_{a_{n}}^{\alpha_{n}} \circ\left(\Sigma_{1} \cap \ldots \cap \Sigma_{m}\right)
$$

where $\Sigma_{i}=\mathcal{F}_{\phi_{i, 1}} \cup \ldots \cup \mathcal{F}_{\phi_{i, m_{i}}}$. Let $F(x)$ be the general $\mathcal{A}$-definable property

$$
F(x) \equiv \bigwedge_{i=1}^{m} \bigvee_{k=1}^{m_{i}} \phi_{i, k}
$$

and let $\succeq_{\left(\alpha_{1}, a_{1}\right) \times \ldots \times\left(\alpha_{n}, a_{n}\right)}$ be the $\mathcal{A}$-definable ordering. Finally, let $x \succeq y$ be the general $\mathcal{A}$-definable preference relation

$$
x \succeq y \equiv \neg F(y) \vee\left(F(x) \wedge x \succeq_{\left(\alpha_{1}, a_{1}\right) \times \ldots \times\left(\alpha_{n}, a_{n}\right)} y\right)
$$

As in Theorem 3.6, we can show that $x \succeq y \Leftrightarrow x \succeq_{P} y$.

First, assuming $x \succeq_{P} y$ holds, i.e. there exists a list $l$ such that

$$
x, y \in l \wedge l^{\prime}=P(l) \wedge\left(y \in l^{\prime} \rightarrow\left(y=l_{j}^{\prime} \wedge x=l_{i}^{\prime} \wedge i<j\right)\right) .
$$

If $\neg F(y)$ then $x \succeq y$ holds trivially and we are done. So let us assume $F(y)$. This means that $y$ passes all the filters of $P$ and hence $y \in l^{\prime}$ and $x \in l^{\prime}$, with $y$ coming later than $x$ on the resulting list. Since the sorting component of $P$ is $\mathcal{S}_{a_{1}}^{\alpha_{1}} \circ \ldots \circ \mathcal{S}_{a_{n}}^{\alpha_{n}}$, and $y$ comes later than $x$ in $l^{\prime}$, it is clear that $x \succeq_{\left(\alpha_{1}, a_{1}\right) \times \ldots \times\left(\alpha_{n}, a_{n}\right)} y$. For the other direction, assume $\neg F(y) \vee\left(F(x) \wedge x \succeq_{\left(\alpha_{1}, a_{1}\right) \times \ldots \times\left(\alpha_{n}, a_{n}\right)} y\right)$. We must show that for some list $l$ containing $x$ and $y$ we have either

$$
y \notin l^{\prime} \quad \text { or } \quad y=l_{j}^{\prime} \wedge x=l_{i}^{\prime} \wedge i<j
$$

where $l^{\prime}=P(l)$. If $y$ does not pass one of the filters of $P$ this is an easy task. So let us assume $F(y)$. Our assumption then gives $F(x)$ and $x \succeq_{\left(\alpha_{1}, a_{1}\right) \times \ldots \times\left(\alpha_{n}, a_{n}\right)} y$. Hence, let $l$ be any list where $x$ is listed before $y$. Since we have $F(x)$ and $F(y)$, we obtain that $x, y \in l^{\prime}$. Assume $x=l_{i}^{\prime}$ and $y=l_{j}^{\prime}$. Finally, since $x \succeq_{\left(\alpha_{1}, a_{1}\right) \times \ldots \times\left(\alpha_{n}, a_{n}\right)} y$, and the sorting operations of $P$ match this lexicographical ordering, we indeed have that $i<j$.

Theorem 5.7 (General completeness) For any general $\mathcal{A}$-definable preference relation $(X, \succeq)$ there is a general $\mathcal{A}$-procedure $P$ : List $X \rightarrow$ List $X$ such that $x \succeq y$ iff $x \succeq_{P} y$.

Proof. Again, the proof is similar to that of Theorem 3.7. Suppose the given general $\mathcal{A}$-definable preference relation is of the form

$$
x \succeq y \equiv F(y) \rightarrow\left(F(x) \wedge x \succeq_{\left(\alpha_{1}, a_{1}\right) \times \ldots \times\left(\alpha_{n}, a_{n}\right)} y\right)
$$

for a general $\mathcal{A}$-definable property

$$
F(x) \equiv \bigwedge_{i=1}^{m} \bigvee_{k=1}^{m_{i}} \phi_{i, k}
$$

Define the procedure

$$
P_{\succeq} \equiv \mathcal{S}_{a_{1}}^{\alpha_{1}} \circ \ldots \mathcal{S}_{a_{n}}^{\alpha_{n}} \circ\left(\Sigma_{1} \cap \ldots \cap \Sigma_{m}\right)
$$

where $\Sigma_{i}=\mathcal{F}_{\phi_{i, 1}} \cup \ldots \cup \mathcal{F}_{\phi_{i, m_{i}}}$. It is easy to verify that $x \succeq y$ iff $x \succeq_{P} y$ (cf. proof of Theorem 3.7). 


\section{Conclusion}

In this paper we show that online decision support system can be used to choose rationally. The offered functionality speeds up the decision process. In Simon's terms, the choice environment contains complexity so that the individual's procedure operating on that environment can be simple.

In the case of simple filter and sorting functionality as in widespread use in online platforms this only works if the type of preferences is restricted. We also show how the operations can be extended so that more general notions of preference relations are captured by filter and sorting functionality.

If one considers current technological developments, it becomes evident that support systems become more expressive (or at least that is the design goal of many companies). In the light of our results, this will lead to individual procedures being simpler while still being able to implement perfectly rational choices.

\section{References}

[1] Andrew Caplin, Mark Dean, and Daniel Martin. Search and satisficing. American Economic Review, 101(7):2899-2922, 2011.

[2] Yuxin Chen and Song Yao. Sequential search with refinement: Model and application with click-stream data. Management Science, 63(12):4345-4365, 2016.

[3] Michael Mandler. Rationality and the speed of decision-making. In Proceedings of the 12th Conference on Theoretical Aspects of Rationality and Knowledge, pages 201-207. ACM, 2009.

[4] Michael Mandler, Paola Manzini, and Marco Mariotti. A million answers to twenty questions : Choosing by checklist. Journal of Economic Theory, 147(1):71-92, 2012.

[5] Yusufcan Masatlioglu and Daisuke Nakajima. Choice by iterative search. Theoretical Economics, 8(3):701-728, 2013.

[6] P. Oliva and P. Zahn. Buying Online - A Characterization of Rational Buying Procedures. ArXiv e-prints, January 2018.

[7] Ariel Rubinstein. Definable preferences: An example1. European Economic Review, 42(3-5):553 560,1998

[8] Ariel Rubinstein and Yuval Salant. A model of choice from lists. Theoretical Economics, 1:3-17, 2006.

[9] Yuval Salant and Ariel Rubinstein. (A,f) : Choice with Frames. Review of Economic Studies, 75:12871296, 2008.

[10] Herbert A. Simon. A behavioral model of rational choice. The Quarterly Journal of Economics, 69(1):99-118, 1955.

[11] Herbert A Simon. Rational choice and the structure of the environment. Psychological review, 63(2):129, 1956.

[12] Peter M Todd and Gerd Gigerenzer. Bounding rationality to the world. Journal of Economic Psychology, 24(2):143 - 165, 2003. The Economic Psychology of Herbert A. Simon. 